\title{
EFECTOS DE UN INUSUAL PERÍODO DE ALTA FRECUENCIA DE HURACANES SOBRE EL BENTOS DE ARRECIFES CORALINOS
}

\author{
Pedro M. Alcolado ${ }^{*}$, Darlenys Hernández-Muñoz ${ }^{1}$,Hansel Caballero ${ }^{2}$, Linnet \\ Busutil $^{1}$, Susana Perera ${ }^{3}$ y Gema Hidalgo ${ }^{1}$
}

\begin{abstract}
RESUMEN
En arrecifes coralinos del suroeste de Cuba, se evaluaron los impactos de la inusitada frecuencia e intensidad de huracanes entre el 2001 y el 2007, y de las enfermedades de corales, ambos factores asociados al cambio climático. En las crestas arrecifales, la reducción de la cobertura de coral duro vivo varió de nula a $21 \%$, mientras que la reducción del diámetro máximo promedio de los corales varió de 16 a $40 \mathrm{~cm}$. En los arrecifes frontales, la reducción de la cobertura de coral varió de nula a 14\%, mientras que la reducción del diámetro máximo promedio varió de nula a $26 \mathrm{~cm}$. Sin embargo, en todos los sitios se observaron grandes cambios en el predominio numérico de las especies. En las crestas, este se desplazó de Acropora palmata y otros corales duros a Millepora complanata, Porites astreoides o Acropora prolifera. En los arrecifes frontales, el cambio ocurrió de la dominancia de Montastraea annularis (complejo de especies), Diploria strigosa y Agaricia agaricites a la de P. astreoides o Siderastrea siderea. Con esos cambios, las crestas están perdiendo efectividad como refugios y como disipadoras del oleaje, a causa de la reducción de complejidad estructural. La densidad del erizo Diadema antillarum se mantuvo insignificante y sin cambio en el sur del Golfo de Batabanó, mientras que se incrementó de manera importante en la cresta de Faro Cazones. Los porcentajes de mortalidad reciente y de incidencia de enfermedades de corales se mantuvieron bajos, lo que sugiere una influencia dominante de los huracanes en la condición de los arrecifes coralinos evaluados.
\end{abstract}

Palabras claves: Cambio climático, huracanes, arrecifes coralinos, enfermedades, Cuba.

\begin{abstract}
In coral reefs of southwestern Cuba, we assessed the impacts from unprecedented frequent and intense hurricanes during the period 2001-2007, and from coral diseases, which are both factors considered to be associated with climate change. At the reef crests, live hard coral cover reduction varied from null to $21 \%$, while maximum average coral diameter reduction varied from 16 to $40 \mathrm{~cm}$. In the fore-reefs, the reduction of coral cover varied from null to $14 \%$, while diameter reduction varied from null to $26 \mathrm{~cm}$. However, at all sites, great shifts in species dominance patterns were observed. In reef crests, numerical dominance of Acropora palmata and other hard corals shifted towards increases of Millepora complanata or, to a lesser extent, of Porites astreoides or Acropora prolifera. At the fore-reefs, the shifts occurred from the dominance of Montastraea annularis complex, Diploria strigosa and Agaricia agaricites,

1 Instituto de Oceanología, Ave. 1ra., No. 18406, Reparto Flores, Playa, La Habana, Cuba.

2 Acuario Nacional, Avenida 1ra. y 60, Playa, La Habana, Cuba.

3 Centro Nacional de Áreas Protegidas, Calle 18, No. 4114, Playa, La Habana, Cuba.

*alcolado@ama.cu
\end{abstract}

Recibido 29-I-2009

Aceptado 23-IX-2009

DOI: http://dx.doi.org/10.15359/revmar.1.4 
towards combined increases of $P$. astreoides or Siderastrea siderea. With those ongoing changes, reef crests are losing effectiveness as refuges and wave dissipators because of reduction of structural complexity. The population density of the sea-urchin Diadema antillarum remained negligible and unchanged in the southern Gulf of Batabanó, while it increased significantly at the Faro Cazones reef crest. Percentages of recent mortality and percentages of disease incidence in corals remained low, suggesting a dominant influence from hurricanes on assessed coral reef decline.

Keywords: Climate change, hurricanes, coral reefs, diseases, Cuba.

\section{INTRODUCCIÓN}

El deterioro de los arrecifes coralinos del mundo, así como su vinculación con el cambio climático es ampliamente reconocido (Spalding, 2004; Wilkinson y Souter, 2008). En el Caribe, la cobertura promedio de coral vivo ha descendido un $80 \%$ en tres décadas (Gardner et al. 2003). Los arrecifes del Golfo de Batabanó no se escapan de esta situación (Alcolado et al. 2001a, b).

Las causas de esta situación mundial son tanto de tipo antropogénicas locales (sedimentación terrígena, contaminación, daño mecánico, estrés térmico industrial y sobrepesca) como de eventos de gran escala asociados al cambio climático mundial (aumento de la temperatura superficial de los océanos y de la concentración de $\mathrm{CO}_{2}$ ), con la consecuente acidificación del mar e incidencia de diversas enfermedades en organismos marinos (Hoegh-Guldberg et al. 2008; Kleypas y Hoegh-Guldberg, 2008). Hoegh-Guldberg et al. (2008) pronostican un aumento de la temperatura del mar de al menos $2^{\circ} \mathrm{C}$ entre el 2050 y el 2100. Por su parte, el Panel Internacional de Cambio Climático pronostica que será de $1.8-4.0^{\circ} \mathrm{C}$ para el 2099 (IPCC, 2007). El aumento de la frecuencia e intensidad de los huracanes, que cada vez más parece estar asociado al cambio climático, particularmente, en la fuerza de sus vientos (IPCC, 2007), es otro factor que ha impactado fuertemente a los arrecifes en el Gran Caribe (Gardner et al. 2005).

Investigaciones de varios autores llevan a concluir que: (1) los corales duros más afectados por los huracanes son Acropora palmata (Lamarck, 1816) y Acropora cervicornis (Lamarck, 1816), y que (2) los efectos sobre los arrecifes coralinos dependen de su frecuencia, intensidad y duración, de las características biológicas y morfológicas de las especies dominantes, la profundidad, la historia ecológica del lugar y la influencia de cualquier factor antropogénico (Glynn et al. 1964; Rogers et al. 1982; Tunnicliffe, 1983; Rogers, 1993; Fong y Lirman, 1995; Lirman y Fong, 1996, 1997a y b; Jordán-Dahlgren y Rodríguez-Martínez, 1998; Lirman, 2000, 2003; Gardner et al. 2005; Gleason et al. 2007).

Llama la atención que en el sexenio 2001-2006 pasaron seis huracanes 
a través o cerca de la zona evaluada, mientras que de cinco sexenios anteriores, en dos se registró uno y ninguno en los restantes. El objetivo de esta investigación fue evaluar la importancia relativa e interacciones del impacto de ese período de inusitada frecuencia de huracanes y de las enfermedades de corales, factores asociados al cambio climático, según IPCC (2007).

Esta evaluación fue realizada en el marco del Programa Nacional de Ciencia y Técnica "Los Cambios Globales y la Evolución del Medio Ambiente Cubano", a través de un subcontrato con la Empresa GEOCUBA Estudios Marinos, como parte del proyecto "Bases Oceanográficas para el Estudio de las Afectaciones del Cambio Global a la Biodiversidad Marina y Costera de Cuba".

\section{MATERIALES Y MÉTODOS}

\section{Antecedentes históricos de los arrecifes evaluados}

En una evaluación realizada en el 2001, se constató que las crestas de los arrecifes del sur del Golfo de Batabanó (cayos Campos, Cantiles y Rico) ya se encontraban muy deterioradas. A pesar de que no se pudieron investigar las causas del deterioro de estos arrecifes, es muy probable que eso haya ocurrido en el corto período de 1988 a 1990. Este criterio se basa en que:

- En mayo de 1988, los arrecifes del área evaluada se encontraban en excelente estado (P. M. Alcolado, obs. pers.). Particularmente, las crestas arrecifales estaban densamente pobladas por Acropora palmata saludables y de gran tamaño.

- El 4 de noviembre del mismo año, el Huracán Gilbert causó un fuerte impacto sobre los arrecifes del sur del área de estudio, principalmente sobre las colonias de A. palmata que quedaron devastadas y presentaban blanqueamiento (M. Ponce y R. Puga, com. pers.).

- En 1989 debió existir una alta probabilidad de blanqueamiento intenso de corales, a juzgar por el análisis de imágenes de temperatura superficial del mar de registros AVRHH del Satélite POES de NOAA de alta resolución (Hayes y Goreau, 2008; T. Goreau, com. pers.).

- En 1991 y 1992 no había condiciones propicias para el blanqueamiento de corales, según los $\mathrm{Hot}$ Spots de NOAA/NESDIS y las series de Reynolds de temperatura superficial del mar elaboradas por S. Cerdeira (inédito).

- En 1993, la gran mayoría de las colonias de $A$. palmata ya estaban muertas, pero no recientemente, a juzgar por su apariencia externa (S. Lorenzo, com. pers.). Por esto último, puede inferirse que la mortalidad tuvo lugar uno o dos años antes de la observación.

Este podría resumirse como un corto período en que aparentemente actuaron de manera conjunta la ac- 
ción devastadora del Huracán Gilbert y el blanqueamiento de corales. Este período coincide con uno de los períodos (1987-1990) reportados por Gardner et al. (2003) como de fuerte tasa de deterioro de los arrecifes coralinos en el Gran Caribe.

Sin embargo, el arrecife de Faro Cazones (este del Golfo de Batabanó; $22.0988 \mathrm{~N},-81.5193 \mathrm{O}$ ), tanto en la cresta como en el arrecife frontal, se encontraba en muy buen estado en marzo del 2001 (Alcolado et al. 2001a). Sin duda, eso respondió en gran parte a su ubicación resguardada en la Ensenada de Cazones.

En el área de estudio, el sexenio de 2001 a 2007 evaluado se caracterizó por el paso de seis huracanes, incluyendo el Huracán Iván, cuyos efectos se sintieron en el sur del área de estudio. De estos, uno tuvo categoría 5 en la escala Zaffir-Simpson (Iván: 2004, muy impactante a pesar de su relativa lejanía al sur), dos de categoría 4 (Michelle: 2001 y Dennis: 2005), uno de categoría 3 (Isidore: 2002) y dos de categoría 2 (Lily: 2002 y Charley: 2004) (Figura 1).

Los eventos de blanqueamiento no evidencian haber causado mortalidad de consideración, a juzgar por los reportes de la Red de Monitoreo Voluntario de Alerta Temprana de Arrecifes Coralinos de Cuba (operada por el Instituto de Oceanología en el marco del Proyecto PNUD/GEF Ecosistema Sabana-Camagüey) y los pronósticos a partir de imágenes satelitales proce- sadas de NOAA/NESDIS (Hot spots y Degree Heating Week).

Por otra parte, los sitios de muestreo no presentaron señales de impactos directos del hombre durante las evaluaciones del 2001 y 2007, aparte de que históricamente no han manifestado indicios de contaminación orgánica, según Lluis-Riera $(1972,1977)$. La única influencia humana significativa en los sitios es la omnipresente actividad de pesca de peces y langostas, que no parece ser tan fuerte como para suponer que ha ejercido un impacto perceptible sobre los arrecifes.

\section{Metodología de la evaluación}

\section{Selección de los sitios de estudio}

En arrecifes coralinos seleccionados del sur y este del Golfo de Batabanó se llevó a cabo una evaluación ecológica rápida, con el fin de analizar los efectos de los huracanes y de las enfermedades de corales, ambos factores asociados al cambio climático (IPCC, 2007), aprovechando como línea base de referencia comparativa un oportuno muestreo realizado en los mismos sitios de muestreo en marzo del 2001 (Alcolado et al. 2001a). Los arrecifes seleccionados fueron los del sur de los cayos Campos, Cantiles, Rosario y Rico (Archipiélago de los Canarreos), y el de Faro Cazones (Ensenada de Cazones), que 
Figura 1

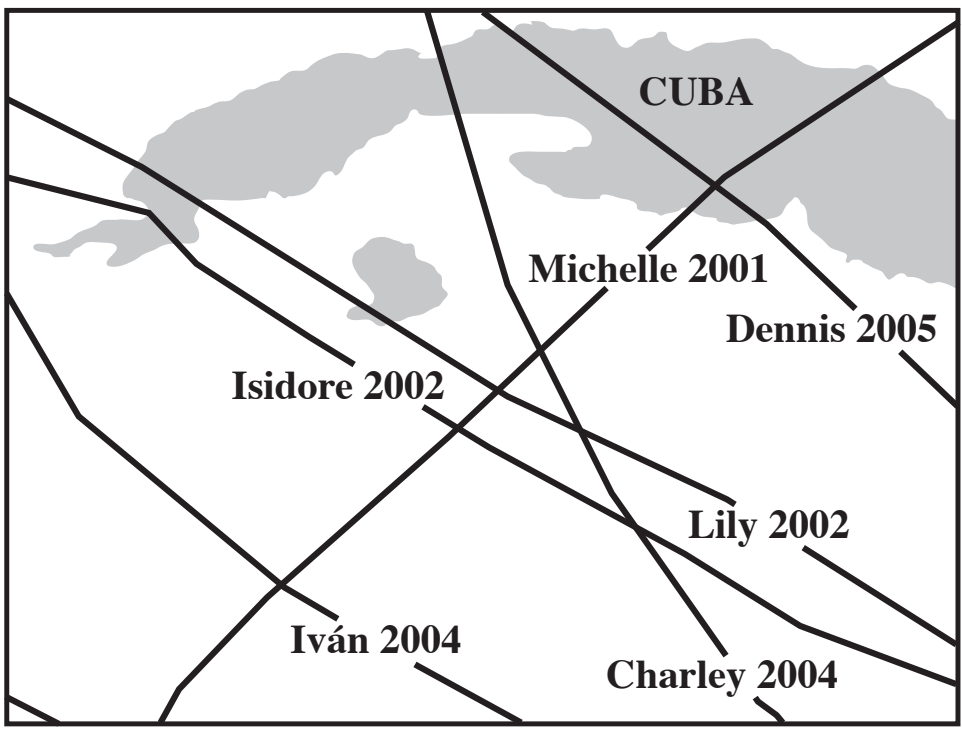

Rutas de los huracanes que pasaron sobre o cerca del área de estudio entre el 2001 y 2007. Modificado de NOAA (http://maps.csc.noaa.gov/hurricanes/viewer.html).

Tracks of hurricanes that passed over or near the study area between 2001 and 2007. Modified from NOAA (http://maps.csc.noaa.gov/hurricanes/viewer.html).

Figura 2

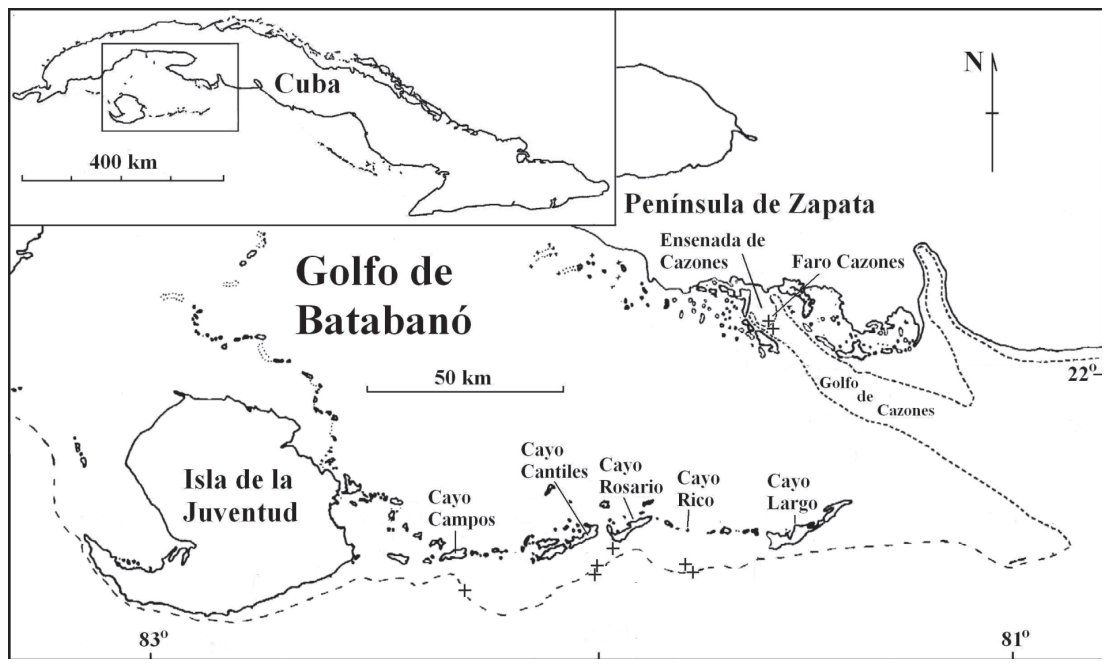

Mapa de ubicación de los sitios de muestreo (indicados con cruces).

Map of sampling site locations (depicted by crosses). 
fueron evaluados entre el 24 y 29 de junio del 2007 (Figura 2).

Para la evaluación se seleccionaron ocho sitios de muestreo (cuatro de crestas arrecifales y cuatro de arrecifes frontales) evaluados en el 2001 (Cuadro 1) con la metodología AGRRA (2000). Estos fueron seleccionados por no mostrar evidencias de haber recibido impactos de origen humano, para evitar lo más posible el ruido de información de origen humano en la percepción de la influencia de factores asociados al cambio climático. Estos arrecifes se encuentran muy alejados y "corriente arriba" de fuentes probables de contaminación. Las actividades humanas se limitan a la pesca y, en algunos casos, al buceo turístico de baja intensidad (menos de 4.000 visitantes/sitio/año).

Indicadores AGRRA y método de muestreo

Cobertura de coral vivo. Porcentaje de coral duro vivo sobre el fondo, intercectado por los transectos. Como corales duros, se incluyeron escleractinios e hidrocorales. Para el cálculo, se descontaron los tramos del transecto ocupados por arena. Las intersecciones se midieron con un tubo de pve de $1 \mathrm{~m}$ de largo, marcado con bandas alternas blancas y negras de $10 \mathrm{~cm}$. Los transectos se distribuyeron al azar a lo largo de 100 m aproximadamente, a casi la misma profundidad $( \pm 1 \mathrm{~m})$.

\section{Cuadro 1}

Ubicación, profundidad y número de transectos de los sitios de muestreo.

Table 1

Location, depth and number of transects of sampling sites.

\begin{tabular}{lccccc}
\hline $\begin{array}{c}\text { Sitio de } \\
\text { muestreo }\end{array}$ & Latitud N & Longitud O & $\begin{array}{c}\text { Profundidad } \\
(\mathbf{m})\end{array}$ & $\begin{array}{c}\text { Número de } \\
\text { transectos } \\
(\mathbf{2 0 0 1})\end{array}$ & $\begin{array}{c}\text { Número de } \\
\text { transectos } \\
(\mathbf{2 0 0 7})\end{array}$ \\
\hline \multicolumn{7}{c}{ Crestas arrecifales } \\
\hline Faro Cazones & 22.0988 & -81.5193 & 0.5 & 8 & 6 \\
Cayo Cantiles & 21.5864 & -81.9573 & 1.4 & 6 & 12 \\
Cayo Rosario & 21.5958 & -81.9272 & 1.3 & 6 & 13 \\
Cayo Rico & 21.5770 & -81.6818 & 1.0 & 6 & 14 \\
\hline & & Arrecifes frontales & & \\
\hline Faro Cazones & 22.0959 & -81.5176 & 10.7 & 6 & 6 \\
Cayo Campos & 21.5488 & -82.3302 & 13.5 & 4 & 12 \\
Cayo Cantiles & 21.5708 & -81.9675 & 11.6 & 6 & 8 \\
Cayo Rico & 21.5734 & -81.6735 & 11.6 & 6 & 16 \\
\hline
\end{tabular}


Diámetro de corales duros. Máxima dimensión de los corales intersectados por los transectos vistos desde arriba (parte superior del eje de crecimiento del coral). Sólo se tuvieron en cuenta los corales con o más de $10 \mathrm{~cm}$ de diámetro máximo. Se empleó el mismo instrumento de medición.

Porcentaje de mortalidad reciente. Porcentaje de área muerta de la superficie de los corales intersectados, que aún conserva la estructura de los cálices y suele estar cubierta, cuando más, por sedimentos y/o por algas y pequeños organismos fijos. Se considera que la muerte ocurrió entre uno y dos años antes del muestreo. El porcentaje fue evaluado igual que la cobertura de coral vivo.

Porcentaje de mortalidad antigua. Porcentaje de área muerta de la superficie de los corales intersectados, que ya no muestra la estructura clara de los cálices; vistos desde arriba como se explicó anteriormente; incluyó los corales muertos que aún se mantenían en pie.

Porcentaje de corales con enfermedades. El porcentaje total de colonias afectadas por alguna enfermedad microbiana (no incluye blanqueamiento).

Densidad de población del erizo negro de espinas largas Diadema antillarum. Se consideró el número de erizos Diadema antillarum (Philippi, 1845) encontrados a lo largo de la banda de $1 \mathrm{~m}$ de ancho en transectos de $10 \mathrm{~m}$ de largo (los mismos usados para cobertura viva de coral).

No se incluyeron el análisis de la abundancia de algas y ni el de reclutas de corales, por ser sus plazos de variación muy diferentes de los efectos de los factores analizados (huracanes, enfermedad de blanqueamiento de corales y enfermedades microbianas de corales).

\section{Procesamiento estadístico}

Para las comparaciones entre el 2001 y el 2007 se utilizó la prueba-t de Student para muestras independientes, con $p=0.05$. Se aplicó transformación de las variables de acuerdo con la Ley de la Potencia de Taylor. Para probar la homogeneidad de varianza se aplicaron las pruebas de Hartley, de Levene y de Brown y Forsythe (STATISTICA v.6). Cuando no hubo homogeneidad de varianza en los datos transformados (datos de mortalidad reciente en crestas y arrecifes frontales, así como de mortalidad antigua en arrecifes frontales), se aplicó la prueba no paramétrica de los signos.

En las comparaciones entre el 2001 y el 2007 de los promedios de los conjuntos de crestas y de arrecifes frontales, se utilizó, respectivamente, la transformación raíz cuadrada de $(\mathrm{x}+1)$ o $\log (\mathrm{x}+1)$, según la simple comparación de la varianza con la media (Elliot, 1977). 
Fuentes complementarias de información

Dadas la extensión y lejanía del área evaluada, la frecuencia de ocurrencia de eventos que afectan a los arrecifes y la necesidad de información sobre estos, se ha recurrido a comunicaciones personales de testigos de efectos de huracanes, que incluyen biólogos marinos y un geógrafo, así como voluntarios de la Red de Monitoreo Voluntario de Alerta Temprana de Arrecifes Coralinos de Cuba (buzos instructores de centros de buceo, especialistas de áreas protegidas, aficionados al buceo y al medio ambiente e investigadores). Esta red comenzó a operar en fase experimental a mediados de 1995 en el marco del Proyecto PNUD/GEF Ecosistema Sabana-Camagüey y se formalizó en el 2004.

\section{RESULTADOS}

\section{Crestas arrecifales}

En las crestas arrecifales, los cambios de la cobertura viva de coral duro no fueron significativos, excepto en Faro Cazones, con una reducción de $21 \%$. Sin embargo, en todos los casos ocurrió un cambio marcado en el patrón de predominio de las especies de corales (Cuadros 2 y 3 ).

En general, la dominancia numérica del coral A. palmata y, en menor grado, la de Porites porites (Pallas, 1766) y el complejo de Montastraea annularis (Ellis y Solander, 1876), decreció en favor de un mayor predominio, según el caso de Porites astreoides (Lamarck, 1816), Acropora prolifera (Lamarck, 1816) y Millepora complanata (Lamarck, 1816), ya sea por separado o en combinaciones. Sin embargo, en dos de los sitios, la representatividad de $P$. porites astreoides disminuyó. Sólo en la cresta de cayo Rosario no hubo cambio importante en el predominio de A. palmata (Cuadro 3).

El diámetro máximo promedio de los corales presentó reducciones significativas en todas las crestas, que variaron entre $16 \mathrm{~cm}$ en Faro Cazones y $40 \mathrm{~cm}$ en cayo Rico (Cuadro 2).

El porcentaje promedio de corales con enfermedades en las crestas en conjunto fue muy bajo y no varió de forma significativa $(\mathrm{p}=0.073611)$, en comparación con el 2001 (de 2.56 $\pm 1.44 \%$ en el 2001 a $0.71 \pm 1.43 \%$ en el 2007). La enfermedad más frecuente fue la viruela blanca. Hubo escasos reportes de banda negra y de banda blanca, pero los de esta última fueron dudosos.

La mortalidad reciente promedio del conjunto de crestas se mantuvo baja y descendió significativamente de $1.74 \pm 0.88 \%$ en el 2001 a 0.18 $\pm 0.23 \%$ en el $2007(\mathrm{p}=0.01415)$. En los sitios de muestreo, la disminución en la mortalidad de los corales fue significativa en los cayos Cantiles, Rosario y Rico (Cuadro 2).

La mortalidad antigua promedio del conjunto de las crestas se man- 


\section{Cuadro 2}

Comparación de indicadores biológicos y significación de la prueba-t de Student por sitios entre los años 2001 y 2007. Se da la media \pm desviación estándar y, entre paréntesis, el número de transectos. El asterisco indica que se aplicó prueba no paramétrica de los signos.

\section{Table 2}

Comparison of biological indicators and significance of Stundent t-test by sites between years 2001 and 2007. Average \pm standard deviation and number of transects (in parentheses) are given. Asterisc indicates that the non-parametric test of signs was applied.

\begin{tabular}{|c|c|c|c|c|}
\hline Sitio & 2001 & 2007 & p & Diferencia \\
\hline \multicolumn{5}{|c|}{ CRESTAS ARRECIFALES } \\
\hline \multicolumn{5}{|c|}{ Cobertura viva de corales duros $(\%)$} \\
\hline Faro Cazones & $70.6 \pm 12.6(6)$ & $49.5 \pm 10.8(6)$ & 0.010170 & Significativa \\
\hline Cayo Cantiles & $15.7 \pm 11.9(6)$ & $9.2 \pm 9.0(12)$ & 0.221576 & No significativa \\
\hline Cayo Rosario & $11.0 \pm 6.2(4)$ & $14.0 \pm 6.3(13)$ & 0.427474 & No significativa \\
\hline Cayo Rico & $24.7 \pm 17.9(6)$ & $23.3 \pm 12.3(14)$ & 0.167554 & No significativa \\
\hline \multicolumn{5}{|c|}{ Diámetro máximo de colonias de corales duros $(\mathrm{cm})$} \\
\hline Faro Cazones & $96.0 \pm 49.5(97)$ & $79.2 \pm 58.4(70)$ & 0.018292 & Significativa \\
\hline Cayo Cantiles & $113.9 \pm 92.8(76)$ & $88.9 \pm 82.8(78)$ & 0.026539 & Significativa \\
\hline Cayo Rosario & $83.1 \pm 66.6(63)$ & $51.8 \pm 50.6(79)$ & 0.000149 & Significativa \\
\hline Cayo Rico & $101.4 \pm 83.8(55)$ & $61.5 \pm 42.3(92)$ & 0.031861 & Significativa \\
\hline \multicolumn{5}{|c|}{ Mortalidad reciente en superficie de colonias de corales duros $(\%)$} \\
\hline Faro Cazones & $1.17 \pm 8.3(94)$ & $0.51 \pm 2.3(70)$ & $0.751830 *$ & No significativa \\
\hline Cayo Cantiles & $0.8 \pm 2.6(62)$ & $0.0(77)$ & $0.041227 *$ & Significativa \\
\hline Cayo Rosario & $4.5 \pm 14.4(56)$ & $0.0(79)$ & $0.002569 *$ & Significativa \\
\hline Cayo Rico & $2.5 \pm 6.5(48)$ & $0.1 \pm 0.7(98)$ & $0.026857 *$ & Significativa \\
\hline \multicolumn{5}{|c|}{ Mortalidad antigua en superficie de colonias de corales duros $(\%)$} \\
\hline Faro Cazones & $13.6 \pm 26.8(97)$ & $13.0 \pm 20.5(70)$ & 0.973841 & No significativa \\
\hline Cayo Cantiles & $54.9 \pm 42.0(74)$ & $42.6 \pm 47.8(78)$ & 0.018367 & Significativa \\
\hline Cayo Rosario & $48.6 \pm 39.5(63)$ & $17.3 \pm 33.7(79)$ & 0.000000 & Significativa \\
\hline Cayo Rico & $33.6 \pm 37.2(55)$ & $17.4 \pm 32.3(98)$ & 0.000283 & Significativa \\
\hline \multicolumn{5}{|c|}{ ARRECIFES FRONTALES } \\
\hline \multicolumn{5}{|c|}{ Cobertura viva de corales duros $(\%)$} \\
\hline Faro Cazones & $25.9 \pm 6.1(6)$ & $11.5 \pm 6.0(6)$ & 0.003360 & Significativa \\
\hline Cayo Cantiles & $20.0 \pm 9.4(6)$ & $6.5 \pm 2.2(8)$ & 0.000300 & Significativa \\
\hline Cayo Rico & $15.5 \pm 8.0(6)$ & $17.2 \pm 8.8(16)$ & 0.831839 & No significativa \\
\hline Cayo Campos & $20.6 \pm 5.6(4)$ & $21.1 \pm 7.5(12)$ & 0.999864 & No significativa \\
\hline \multicolumn{5}{|c|}{ Diámetro máximo de colonias de corales duros (cm) } \\
\hline Faro Cazones & $42.1 \pm 37.8(103)$ & $27.3 \pm 15.7(104)$ & 0.000007 & Significativa \\
\hline Cayo Cantiles & $24.3 \pm 12.6(93)$ & $23.7 \pm 12.8(31)$ & 0.457333 & No significativa \\
\hline Cayo Rico & $31.4 \pm 20.8(59)$ & $34.6 \pm 31.4(104)$ & 0.502758 & No significativa \\
\hline Cayo Campos & $54.3 \pm 58.3(68)$ & $28.6 \pm 19.1(162)$ & 0.000019 & Significativa \\
\hline
\end{tabular}




\begin{tabular}{|c|c|c|c|c|}
\hline Sitio & 2001 & 2007 & $\mathbf{p}$ & Diferencia \\
\hline \multicolumn{5}{|c|}{ ARRECIFES FRONTALES (Continuación) } \\
\hline \multicolumn{5}{|c|}{ Mortalidad reciente en superficie de colonias de corales duros (\%) } \\
\hline Faro Cazones & $0.9 \pm 4.6(103)$ & $0.4 \pm 2.4(104)$ & $0.342782 *$ & No significativa \\
\hline Cayo Cantiles & $2.2 \pm 10.9(93)$ & $0.0(31)$ & No da valor* & No significativa \\
\hline Cayo Rico & $1.0 \pm 3.4(60)$ & $0.0(104)$ & $0.023342 *$ & Significativa \\
\hline Cayo Campos & $6.0 \pm 12.1(68)$ & $2.3 \pm 12.9(162)$ & $0.000022 *$ & Significativa \\
\hline \multicolumn{5}{|c|}{ Mortalidad antigua en superficie de colonias de corales duros (\%) } \\
\hline Faro Cazones & $26.4 \pm 29.1(103)$ & $15.0 \pm 23.8(104)$ & $0.030059^{*}$ & Significativa \\
\hline Cayo Cantiles & $14.6 \pm 24.2(93)$ & $27.4 \pm 31.8(31)$ & $0.044171 *$ & Significativa \\
\hline Cayo Rico & $33.6 \pm 37.7(60)$ & $17.4 \pm 32.3(104)$ & $0.000000 *$ & Significativa \\
\hline Cayo Campos & $35.9 \pm 34.2(68)$ & $8.6 \pm 22.5(162)$ & $0.000000^{*}$ & Significativa \\
\hline
\end{tabular}

\section{Cuadro 3}

Porcentaje de colonias de las especies de corales duros en los sitios de cresta en el 2001 y 2007.

\section{Table 3}

Percentage of colonies of stony coral species at crest sites in 2001 and 2007.

\begin{tabular}{|c|c|c|c|c|c|c|c|c|}
\hline \multirow{2}{*}{ Especies } & \multicolumn{2}{|c|}{ Faro Cazones } & \multicolumn{2}{|c|}{ Cayo Cantiles } & \multicolumn{2}{|c|}{ Cayo Rosario } & \multicolumn{2}{|c|}{ Cayo Rico } \\
\hline & 2001 & 2007 & 2001 & 2007 & 2001 & 2007 & 2001 & 2007 \\
\hline Acropora palmata & 84 & 57 & 35 & 2 & 19 & 23 & 51 & 17 \\
\hline Acropora prolifera & 10 & 27 & 0 & 0 & 0 & 0 & 0 & 0 \\
\hline Agaricia agaricites & 0 & 0 & 0 & 2 & 0 & 0 & 0 & 0 \\
\hline Diploria clivosa & 0 & 0 & 6 & 0 & 0 & 1 & 0 & 0 \\
\hline Diploria strigosa & 0 & 0 & 8 & 2 & 0 & 1 & 0 & 0 \\
\hline Isophyllastrea rigida & 0 & 0 & 6 & 0 & 0 & 0 & 0 & 0 \\
\hline Manicina areolata & 1 & 0 & 0 & 0 & 0 & 0 & 0 & 0 \\
\hline Millepora complanata & 5 & 1 & 8 & 15 & 4 & 56 & 4 & 73 \\
\hline $\begin{array}{l}\text { Montastraea annularis } \\
\text { (complejo de especies) }\end{array}$ & 0 & 3 & 4 & 6 & 19 & 0 & 8 & 0 \\
\hline Montastraea cavernosa & 0 & 0 & 0 & 0 & 2 & 0 & 0 & 0 \\
\hline Porites astreoides & 0 & 11 & 8 & 64 & 34 & 18 & 24 & 11 \\
\hline Porites divaricata & 0 & 0 & 0 & 2 & 0 & 0 & 0 & 0 \\
\hline Porites porites & 0 & 0 & 24 & 6 & 17 & 0 & 9 & 0 \\
\hline Siderastrea siderea & 0 & 0 & 0 & 0 & 4 & 0 & 2 & 0 \\
\hline
\end{tabular}


tuvo moderada y varió de $37.7 \% \pm$ $18.3 \%$ en el 2001 a $22.6 \% \pm 13.5 \%$ en el 2007, pero no de forma significativa $(p=0.248124)$. En los sitios de muestreo, la disminución fue significativa en los cayos Cantiles, Rosario y Rico (Cuadro 2).

La densidad de Diadema antillarum se mantuvo muy baja en el conjunto de las crestas $(0.3 \pm 0.6$ ind./ $\mathrm{m}^{2}$ en el 2001 y $0.9 \pm 1.8$ ind. $/ \mathrm{m}^{2}$ en el 2007) y no mostró diferencias significativas entre los dos años $(\mathrm{p}=$ 0.8116218). Sin embargo, en los sitios hubo un incremento importante en la cresta de Faro Cazones (1.17 ind. $/ \mathrm{m}^{2}$ en el 2001 a 3.6 ind. $/ \mathrm{m}^{2}$ en el 2007), aunque este no fue estadísticamente significativo $(\mathrm{p}=0.515376)$, probablemente debido a error Tipo II.

\section{Arrecifes frontales}

En los arrecifes frontales, la reducción de la cobertura viva de coral varió entre estadísticamente no significativa (cayos Rico y Campos) y 14\% (Faro Cazones). Sólo en Faro Cazones y cayo Cantiles se observaron reducciones significativas (Cuadro 2).

Sin embargo, igual que en las crestas, en todos los sitios se observaron notables cambios en el patrón de predominio de las especies. El predominio de uno u otro de los corales M. annularis (como complejo de especies), Diploria strigosa (Houttuyn, 1772) y Agaricia agaricites (Lamarck, 1758) se vio desplazado hacia el aumento (en distintas combinaciones) de $P$. as- treoides y Siderastrea siderea (Ellis y Solander, 1786) (Cuadro 4). En el complejo de M. annularis hubo un incremento del porcentaje de $M$. faveolata (Ellis y Solander, 1786) en los cayos Cantiles y Campos (de 9\% a 23\% y de $6 \%$ a $23 \%$, respectivamente).

La reducción del diámetro máximo promedio fue significativa sólo en Faro Cazones y en cayo Campos (Cuadro 2). El porcentaje promedio de corales con enfermedades del conjunto de arrecifes frontales se mantuvo muy bajo $(0.35 \pm 0.44 \%$ en el 2001 y $0.14 \pm 0.29 \%$ en el 2007$)$ y sin diferencia significativa $(p=0.447904)$ en el 2007. Las enfermedades más frecuentes fueron la mancha oscura y la plaga blanca. Unas pocas fueron reportadas como desconocidas.

La mortalidad reciente promedio del conjunto de arrecifes frontales descendió de $2.5 \pm 2.4 \%$ a $0.7 \% \pm$ $1.1 \%$, pero de manera no significativa $(p=0.147389)$. A pesar de la no significación de las diferencias, es notable la frecuencia mucho mayor de porcentajes cero en el 2007. En los sitios, esta reducción sí fue significativa en los cayos Rico y Campos (Cuadro 2).

En el conjunto de sitios de arrecifes frontales, el promedio de mortalidad antigua tampoco cambió de manera significativa $(24.4 \% \pm 9.0 \%$ en el $2001 \mathrm{y}$ $14 \% \pm 9.0 \%$ en el $2007 ; p=0.156360$ ), lo que puede deberse a un error estadístico Tipo II. Sin embargo, la comparación en los sitios mostró disminuciones significativas en Faro Cazones y en los cayos Rosario y Rico. De forma con- 


\section{Cuadro 4}

Porcentaje de colonias de las especies de corales duros en los sitios de arrecife frontal en el 2001 y 2007. Entre paréntesis (en la columna de los años) se da el número de especies incluidas en el porcentaje de otras especies que no se mencionan en aras de la brevedad, por no ser pertinentes a este análisis.

\section{Table 4}

Percentage of colonies of stony coral species at fore-reef sites in 2001 and 2007. In parentheses (in the columns of years), the number of species included in the percentages of other species are given, but are not mentionned in the sake of brevity because they are not relevant to this analysis.

\begin{tabular}{|c|c|c|c|c|c|c|c|c|}
\hline \multirow[t]{2}{*}{ Especies } & \multicolumn{2}{|c|}{ Faro Cazones } & \multicolumn{2}{|c|}{$\begin{array}{c}\text { Cayo } \\
\text { Cantiles }\end{array}$} & \multicolumn{2}{|c|}{ Cayo Rico } & \multicolumn{2}{|c|}{ Cayo Campos } \\
\hline & 2001 & 2007 & 2001 & 2007 & 2001 & 2007 & 2001 & 2007 \\
\hline Agaricia agaricites & 6 & 7 & 7 & 3 & 25 & 9 & 9 & 2 \\
\hline Diploria labyrinthiformis & 0 & 0 & 0 & 0 & 0 & 0 & 6 & 0 \\
\hline Diploria strigosa & 0 & 0 & 10 & 7 & 5 & 4 & 8 & 1 \\
\hline Madracis decactis & 0 & 0 & 0 & 7 & 0 & 0 & 0 & 0 \\
\hline $\begin{array}{l}\text { Montastraea annularis } \\
\text { (complejo de especies) }\end{array}$ & 37 & 24 & 33 & 23 & 12 & 19 & 33 & 33 \\
\hline Montastraea cavernosa & 6 & 6 & 4 & 7 & 7 & 0 & 5 & 10 \\
\hline Porites astreoides & 9 & 21 & 10 & 7 & 5 & 14 & 8 & 19 \\
\hline Porites porites & 6 & 2 & 0 & 0 & 14 & 11 & 8 & 3 \\
\hline Siderastrea siderea & 17 & 30 & 17 & 37 & 27 & 35 & 12 & 14 \\
\hline Stephanocoenia intercepta & 0 & 0 & 0 & 0 & 0 & 0 & 0 & 6 \\
\hline Otras especies $(<5 \%)$ & $\begin{array}{c}17 \\
(8 \\
\text { spp.) }\end{array}$ & $\begin{array}{c}11 \\
(7 \\
\text { spp.) }\end{array}$ & $\begin{array}{c}18 \\
(9 \\
\text { spp.) }\end{array}$ & $\begin{array}{c}6 \\
(2 \\
\text { spp. })\end{array}$ & $\begin{array}{c}6 \\
(3 \\
\text { spp.) }\end{array}$ & $\begin{array}{c}8 \\
(3 \\
\text { spp.) }\end{array}$ & $\begin{array}{c}11 \\
(5 \\
\text { spp.) }\end{array}$ & $\begin{array}{c}10 \\
(6 \\
\text { spp.) }\end{array}$ \\
\hline
\end{tabular}

traria, en cayo Cantiles ocurrió un aumento significativo de la mortalidad antigua, como era de esperarse ante impactos de huracanes (Cuadro 2).

En los arrecifes frontales como un todo, la densidad de D. antillarum se mantuvo muy baja $\left(0.0\right.$ ind. $/ \mathrm{m}^{2}$ en el 2001 y $0.05 \pm 0.1 \mathrm{ind} . / \mathrm{m}^{2}$ en el
2007) y sin cambio significativo $(\mathrm{p}=$ $0.355918)$.

\section{DISCUSIÓN}

El hecho de que todos los sitios, incluso los que no sufrieron reducciones significativas en la co- 
bertura viva de coral, experimentaron marcados cambios en el predominio numérico de especies indica que la conservación de la cobertura por sí sola no necesariamente representa estados estables integrales y favorables de los arrecifes coralinos.

En las crestas, M. complanata es una de las especies que incrementó considerablemente su predominio. Este hidrocoral es de crecimiento rápido, muy resistente al oleaje y por su forma es poco afectado por la sedimentación, lo que lo hace un invasor exitoso del espacio disponible (Lewis, 1996), en detrimento de A. palmata en las crestas arrecifales. Eso puede restringir las posibilidades de recuperación del espacio por esta última especie, que es la principal constructora de crestas y la de mayor importancia para mantener la complejidad estructural, disipar la energía del oleaje y brindar refugio a diversos organismos.

$S$. siderea y $P$. astreoides, que también incrementaron su predominio, son muy resistentes a la sedimentación y al oleaje (Hubbard y Pocock, 1972; Kolehmainen, 1973; Lirman y Fong, 1996; Torres y Morelock, 2002). En particular, P. astreoides ha sido señalada como una especie con gran potencial reproductivo y comparativamente poco dañada por los huracanes (Glynn et al. 1964; Lirman y Fong, 1996 y 1997b). Ambas especies apenas contribuyen al relieve de la cresta y, por lo tanto, no son tan aptas como refugio, sobre todo la última, por su hábito que tiende a ser incrustante.

El aumento del predominio numérico de A. prolifera en la cresta de Faro Cazones puede explicarse por su crecimiento rápido y capacidad de reproducirse por fragmentación (Gladfelter et al. 1978; Acropora Biological Review Team, 2005). Si bien brinda abundante refugio con sus ramas, estas no tienen el grosor ni la fortaleza de A. palmata para garantizar la integridad a largo plazo de una cresta arrecifal sometida a frecuentes huracanes. Atendiendo a la cobertura aún elevada de A. palma$t a$, sólo en este sitio esta especie parece conservar el potencial de recuperar con relativa rapidez el predominio inicial, si una menor frecuencia e intensidad de huracanes lo permite.

En el sitio de cresta de Faro Cazones fue evidente el gran impacto observado inmediatamente después del paso del Huracán Michelle en el 2001, a manera de un gran destrozo y dispersión de las ramas partidas del coral A. palmata, y de corales Diploria volcados. Después del paso del Huracán Dennis en el 2005 no se observó tanto daño, pero sí un enturbiamiento muy fuerte del agua de color verdoso (J. González, com. pers.). Es conveniente aclarar que este último huracán ejerció su influencia no desde el mar, sino desde tierra firme. Por otro lado, el impacto de los huracanes fue más notorio en Faro Cazones, casi seguramente debido a que este sitio se encontraba en muy buen estado antes de ser impactado en el 
2001 por Michelle, mientras que los sitios restantes, ya estaban muy deteriorados. Al respecto, Lirman y Fong (1996) refieren como un arrecife sobre el cual no ha pasado un huracán en muchos años y ha desarrollado una estructura compleja, como el de Discovery Bay, Jamaica, es más vulnerable que uno sobre el cual pasan huracanes con mayor frecuencia.

Al parecer, la cobertura de corales en los arrecifes frontales de Faro Cazones y cayo Campos no disminuyó gracias a la ubicación muy poco expuesta del primer sitio y la mayor lejanía del segundo con respecto a las trayectorias de los huracanes más fuertes; aparte de estar ambos sitios a mayor profundidad que los de cresta. El hecho de que tanto en las crestas como en los arrecifes frontales, en la mayoría de los sitios la mortalidad antigua promedio haya tendido a disminuir contrariamente a lo esperado, podría explicarse por una contribución de tejido vivo por reclutamiento y por crecimiento de colonias preexistentes.

Los huracanes Michelle (2001), Isidore (2002), Lily (2002) y Charley (2004) pasaron muy cerca de los tres sitios al sur del Golfo de Batabanó, que como se explicó ya estaban muy afectados cuando se evaluaron en el 2001. El Huracán Iván (2004), a pesar de su paso relativamente distante, generó un oleaje muy fuerte en esa área (I. Mitrani, Instituto de Meteorología, com. pers.), lo que debe de haber causado una fuerte afectación a los arrecifes de los cayos Campos,
Cantiles, Rosario y Rico, sobre todo en las crestas, como reflejan nuestros resultados. El más afectado fue el de cayo Cantiles, el único donde el porcentaje de mortalidad antigua aumentó. A ello debe de haber contribuido, además del oleaje, una fuerte sedimentación, a juzgar por las plumas de sedimentos que suelen salir por el canal de cayo Rosario cuando el oleaje se intensifica, como sucede durante el paso de huracanes.

Observaciones de campo en 1987 y 1988 (P. M. Alcolado, obs. pers.) han revelado que estas plumas de sedimentos suelen presentar una dirección hacia el oeste y suroeste, por lo que el arrecife de cayo Rosario, al este del canal, debe ser algo menos afectado, más bien de forma marginal, que los que están al oeste. Esto también se puede observar claramente en imágenes satelitales de Acker et al. (2004), donde la pluma de sedimentos causada por el Huracán Michelle cubrió el sur de cayo Cantiles y de los que están al oeste, incluyendo cayo Campos al extremo oeste del área evaluada. En estas imágenes (tomadas el 5 y el 9 de noviembre del 2001, después de su paso el 4 de noviembre) no se evidencia una fuerte carga de sedimentos en Faro Cazones ni en cayo Rico. Estas imágenes sugieren que los arrecifes entre los cayos Rosario y Campos fueron afectados, no sólo por el fuerte oleaje, sino también por una intensa sedimentación, a diferencia del arrecife de Faro Cazones. En este últi- 
mo, la afectación debe de haber sido principalmente por el oleaje (aunque amortiguado por la posición resguardada de ese arrecife, J. González, com. pers.). Sin embargo, el área de Faro Cazones sí fue afectada por una fuerte sedimentación producida por escorrentía cuando el Huracán Dennis en el 2005 influenció desde tierra firme, como también fue comprobado in situ (J. González, com. pers.).

Es ampliamente reconocido que los huracanes son beneficiosos para los arrecifes si no son muy frecuentes, ya que impiden que el espacio sea monopolizado por especies de coral competitivamente más aptas (Rogers, 1993) y permiten la extensión del área de arrecife a través de la propagación y reimplantación de fragmentos vivos de corales (Lirman, 2003).

Según Lirman (2003), a partir de sus experiencias en el sur de la Florida, para el coral $A$. palmata resulta beneficioso el paso de un huracán cada 5 años, aproximadamente. Si la frecuencia es de cada 2 años aproximadamente o menos, como ha sido el caso de nuestra área evaluada, las crestas formadas por ese coral se van degradando sostenidamente al no haber margen de tiempo para recuperaciones sucesivas. Si la frecuencia es de un huracán cada 15 años, el desarrollo de la población del coral se hace más lento.

Durante el período evaluado, no hubo evidencias de eventos de blanqueamiento que hayan causado mortalidad de consideración, a juzgar por los reportes de la Red de Monitoreo Voluntario de Alerta Temprana de Arrecifes Coralinos de Cuba, por los pronósticos de imágenes satelitales de NOAA/NESDIS (Degree Heating Week: DHW) y los datos de mortalidad reciente y antigua de la presente evaluación. De la muy baja mortalidad reciente observada en el 2007, se deduce que al menos el blanqueamiento del 2006 (que se reportó como de bajo a moderado por la mencionada red) no causó afectación de importancia.

Por otro lado, el menor valor o no cambio del porcentaje de mortalidad reciente (considerándola como la ocurrida entre uno y dos años antes del muestreo) en los arrecifes frontales indica que tampoco parece haber ocurrido una mortalidad importante por el blanqueamiento del 2005, a pesar de haber sido fuerte, sobre todo si se tiene en cuenta que incluso la mortalidad antigua fue predominantemente menor en el 2007.

En el 2004 no se reportó blanqueamiento de significación. Por otro lado, en el 2003 el blanqueamiento tendió a ser de nulo a débil en la costa sur de Cuba, mientras que en el 2002 fue pobre en la relativamente cercana Bahía de Cochinos, al este. De este modo, puede decirse que la enfermedad de blanqueamiento, como fenómeno inherente al cambio climático, no ha mostrado evidencia de haber tenido un impacto letal de consideración como el de los huracanes en el área durante el sexenio evaluado. 
Si bien los porcentajes de corales afectados por enfermedades microbianas fueron bajos y mostraron disminuciones locales, ello no deja de preocupar, debido a que estas pueden retrasar en cierta medida, junto con otros factores, la tasa con que las comunidades de corales se recuperan de los impactos de los huracanes, lo que podría ser más preocupante si se mantuviera una tendencia de altas frecuencias e intensidades de estos.

Al respecto, Gardner et al. (2005), en un metaanálisis del impacto de los huracanes sobre los arrecifes coralinos del Gran Caribe desde 1980, concluyen que los huracanes produjeron una mortalidad promedio de $6 \%$ de los corales, mientras que el conjunto de otros factores, incluyendo las enfermedades, fue de $2 \%$. Si consideramos que los corales detectados con muerte reciente murieron dentro del plazo de un año aproximadamente, nuestras estimaciones del 2001 $(1.74 \%$ en crestas y $2.5 \%$ en arrecifes frontales) son muy cercanas a las de esos autores. Eso no ocurrió así en el año 2007, cuando los porcentajes fueron significativamente muy inferiores $(0.18 \%$ en crestas y $0.7 \%$ en arrecifes frontales), lo que podría implicar un pronóstico aparentemente más alentador. No obstante, desconocemos cómo se comportaron los años intermedios del período 2001-2007, por lo que se necesita un monitoreo sistemático de este indicador, con el fin de esclarecer su tendencia.
En la literatura hay indicios de que los corales, a través de cambios en la composición de sus zooxantelas simbióticas y de su biota microbiana, parecen estar adaptándose al blanqueamiento (Baker, 2001) y a las enfermedades de origen microbiano (Reshef et al. 2006; Lesser et al. 2007). Esto sugiere un escenario futuro más favorable, aun con los cambios no deseables que se están produciendo en el patrón de predominio de especies de corales. No obstante, debe tenerse en cuenta que si sigue aumentando la temperatura del agua, se llegaría a la muerte fisiológica de los corales (Schuttenberg y Marshall, 2008) sin mediación de enfermedades ni de blanqueamiento. Por ello, siempre será crucial contribuir en lo posible a detener cuanto antes el calentamiento mundial.

La baja densidad de $D$. antillarum se mantiene como un problema en detrimento de la potencialidad de la recuperación de las comunidades de corales después de huracanes (Rogers, 1993). Esta es una especie clave en el control del desarrollo excesivo de macroalgas bentónicas que compiten por el espacio, que sufrió una catastrófica mortalidad en 1983-1984 en todo el Gran Caribe a causa de una enfermedad desconocida (Lessios et al. 1984). Para que la densidad de esta especie sea efectiva en la remoción de algas debe ser mayor que 2 ind./m², según CARICOMP (2000), por lo que en los sitios evaluados esta especie no debe estar ejerciendo esa 
función, excepto en la cresta de Faro Cazones.

El arrecife de coral de Faro Cazones (tanto en la cresta como en el arrecife frontal) sigue siendo el de mejor condición integral y, por lo tanto, puede ser considerado como el más "resiliente" en un sentido amplio (o sea, no referido exclusivamente al blanqueamiento de corales). Esto se refuerza por el hecho de que en la evaluación del 2001, este arrecife se encontraba en muy buen estado luego de haber sufrido el embate muy cercano del Huracán Lily (categoría 2) en 1996, lo que muestra su gran capacidad de recuperación.

Por esa "resiliencia", el arrecife de Faro Cazones merece mantenerse protegido por el Sistema Nacional de Áreas Protegidas (dentro del Parque Nacional Ciénaga de Zapata) como fuente de propágulos para la recuperación de otros arrecifes, cuyas poblaciones de corales duros se encuentran diezmadas. De hecho, la cresta aún presenta deseables características pleistocénicas de dominancia de A. palmata. Esto puede atribuirse en gran medida al carácter resguardado que disfruta en la Ensenada de Cazones (norte del Golfo de Cazones), donde, además, según los mapas de distribución de nutrientes de Fernández de la Llera et al. (1990), las aguas son más fértiles y, por lo tanto, más productivas. Esto propiciaría la alimentación heterotrófica de los corales y que al haber menos trans- parencia se filtre parte de la luz durante los eventos de calentamiento, lo que según Schuttenberg y Marshall (2008) reduce la vulnerabilidad al blanqueamiento y agregamos que favorece la recuperación ante impactos de otro tipo.

\section{CONCLUSIONES}

1. Durante el período 2001-2007, los huracanes fueron el factor asociado al cambio climático, que más afectó a los arrecifes evaluados. El blanqueamiento y las enfermedades infecciosas de corales tuvieron una repercusión menor que la esperada.

2. Coincidiendo con diversos autores, el impacto causado por los huracanes en el período evaluado se comportó de forma muy heterogénea en los sitios, en dependencia de la profundidad, grado de exposición, afectación por sedimentos y estado previo de los arrecifes.

3. Las reducciones de la cobertura viva de coral variaron de no significativa a $21 \%$. Sin embargo, todos los sitios experimentaron cambios en el patrón de predominio de especies en favor de otras más oportunistas (tolerantes a la sedimentación y/o al oleaje, y de crecimiento más rápido). Por lo tanto, la conservación de la cobertura por sí sola no necesariamente representa estados persistentes de integridad biológica en los arrecifes coralinos.

4. Con los cambios en las especies dominantes, las crestas arrecifales están perdiendo efectividad como 
refugio para peces e invertebrados y como disipadoras del oleaje, a causa de la pérdida de $A$. palmata.

5. El arrecife de Faro Cazones sigue siendo el que mejor condición presenta y, por lo tanto, puede ser considerado el más "resiliente".

\section{AGRADECIMIENTOS}

Agradecemos al Programa Nacional de Ciencia y Técnica "Los Cambios Globales y la Evolución del Medio Ambiente Cubano", a la Empresa GEOCUBA Estudios Marinos y al Instituto de Oceanología, por proveer los fondos para la ejecución de esta investigación. Nuestra gratitud también va dirigida a José Espinosa y a los buzos Jorge Oliva, Jorge Oliva Jr. y Alexys Morales, por su ayuda incondicional en el muestreo, así como a Rodolfo Claro Madruga, María Elena Miravet, Margarita Lugioyo, Aida Hernández-Zanuy y Elisa Eva García-Rivera, por la revisión del trabajo y sus valiosos comentarios.

\section{BIBLIOGRAFÍA}

Acker, J. G., Vasilkov, A., Nadeau, D. \& Kuring, N. (2004). Use of SeaWiFS ocean color data to estimate neritic sediment mass transport from carbonate platforms for two hurricane-forced events. $\mathrm{Co}$ ral reefs, 23(1), 39-47.

Acropora Biological Review Team (2005). Atlantic Acropora Status Review Document. Report to
National Marine Fisheries Service, Southeast Regional Office. March 3.

AGRRA (2000). The AGRRA Rapid Assessment Protocol. Atlantic and Gulf Rapid Reef Assessment methodology. Recuperado en junio 25,2001 , disponible en http:// coral.aoml.noaa.gov/agra/methodhome.htm.

Alcolado, P. M., Ginsburg, R. N., Kramer, P., Kosminin, V., de la Guardia, E., González, S. \& Hernández, M. (2001a). Estado de salud del bentos de los arrecifes coralinos del sur y este del Golfo de Batabanó: Informe Preliminar de la Expedición CUBAGRRA. Marzo. (Informe final). Archivo del Instituto de Oceanología, Academia de Ciencias de Cuba.

Alcolado, P. M., Claro-Madruga, R., Martínez-Daranas, B., Menéndez-Macía, G., García-Parrado, P., Cantelar, K., Hernández, M. \& del Valle, R. (2001b). Evaluación ecológica de los arrecifes coralinos del oeste de Cayo Largo del Sur, Cuba: 1998-1999. Bol. Mar. Coas. Res., 30, 109-132.

Baker, A. C. (2001). Ecosystems: Reef corals bleach to survive change. Nature, 411, 765-766.

CARICOMP (2000). Status and temporal trends at CARICOMP coral reef sites. Trabajo presentado en el $9^{\text {th }}$ Int. Coral Reef Symp., Junio, Panamá.

Elliot, J. M. (1977). Some methods for the statistic analysis of sam- 
ples of benthic invertebrates. Freshwater Biological Association. Sci. Publ., 25, 1-156.

Fernández de la Llera, M., Hidalgo-González, R. M., López-García, D., García-Ramil, I. \& Penié-Rodríguez, I. (1990). Caracterización Hidroquímica de la Fosa de Jagua y de la Zona Económica Exclusiva al Sur de Cuba (Informe final de tema). Instituto de Oceanología, Academia de Ciencias de Cuba.

Fong, P. \& Lirman, D. (1995). Hurricanes cause population expansion of the branching coral Acropora palmata (Scleractinia): wound healing and growth patterns of asexual recruits. Mar. Ecol., 16, 317-335.

Gardner, T. A., Cote, I. M., Gill, J. A., Grant, A. \& Watkinson, A. R. (2003). Long term region-wide declines in the Caribbean corals. Sci., 301, 958-960.

Gardner, T. A., Cote, I. M., Gill, J. A., Grant, A. \& Watkinson, A. R. (2005). Hurricanes and Caribbean Reefs: Impacts, recovery patterns and role in long term decline. Ecol., 86(1), 174-184.

Gladfelter, E. H., Monahan, R. K. \& Gladfelter, W. B. (1978). Growth rates of five reef-building corals in the northeastern Caribbean. Bul. Mar. Sci., 28, 728-734.

Gleason, A. C. R., Lirman, D., Williams, D., Gracias, N. R., Gintert, B. E., Madjidi, H., Reid, R. P., Boynton, G. C., Negahdaripour, S. \& Miller, M. (2007).
Documenting hurricane impacts on coral reefs using two-dimensional video-mosaic technology. Mar. Ecol., 28, 254-258.

Glynn, P. W., Almodóvar, L. R. \& González, J. G. (1964). Effects of Hurricane Edith on marine life in La Parguera; Puerto Rico. J. Caribbean Sci., 4(2,3), 335-345.

Hayes, R. \& Goreau, T. J. (2008). An analysis of satellite-derived sea surface temperatures from Caribbean and Atlantic reef sites. 19822003. Rev. Biol. Trop., 56, 97-118. Hoegh-Guldberg, O., Mumby, P. J., Hooten, A. J., Stenek, R. S., Greenfield, P., Gómez, E., Harvell, C. D., Sale, P. F., Edwards, A. J., Kaldeira, K., Knowlton, N., Eakin, C. M., Iglesias-Prieto, R., Muthinga, N., Bradbury, R. H., Duby, A. \& Hatziolos, M. E. (2008). Coral reefs under rapid climate change and Ocean acidification. Sci., 318, 1737-1742.

Hubbard, J. A. \& Pocock, Y. P. (1972). Sediment rejection by recent scleractinian corals: A key to paleoenvironmental reconstruction. Geol. Rundschuge, 61, 598626.

IPCC. (2007). Climate Change 2007: IPCC fourth Assessment Report. Working Group I Report "The Physical Science Basis" IPCC, Geneva, Switzerland.

Jordán-Dahlgren, E. \& Rodríguez-Martínez, R. E. (1998). Post hurricane initial recovery of Acropora palmata in two reefs of 
the Yucatán Península. México. Bul. Mar. Sci., 63(1), 213-228.

Kleypas, J. \& Hoegh-Guldberg, O. (2008). Coral reefs and climate change: susceptibility and consequences. En: C. Wilkinson \& D. Souter (eds.). Status of Caribbean Coral Reefs after Bleaching and Hurricanes in 2005 (pp. 19-29). Global Coral Reef Monitoring Network and Rainforest Research Center, Townsville.

Kolehmainen, S. E. (1973). Siltation experiments on corals in situ. Puerto Rico. Final Report Mission 8. PRINUL.

Lesser, M. P., Bythell, J. C., Gates, R. D., Johnstone, R. W. \& Hoegh-Guldberg, O. (2007). Are infectious diseases really killing corals? Alternative interpretations of the experimental and ecological data. J. Exp. Mar. Biol. Ecol., 346, 36-44.

Lessios, H. A., Robinson, D. R. \& Cubit, J. D. (1984). Spread of Diadema mass mortality throughout the Caribbean. Sci., 226, 335-337.

Lewis, J. B. (1996). Spatial distribution of the calcareus hydrozoans Millepora complanata and Millepora squarrosa on coral reefs. Bul. Mar. Sci., 59(1), 188-195.

Lirman, D. (2000). Fragmentation in the branching coral Acropora palmata (Lamarck): growth, survivorship and reproduction of colonies and fragments. J. Exp. Mar. Biol. Ecol., 251, 41-57.
Lirman, D. (2003). A simulation model of the population dynamics of the branching coral Acropora palmata: effects of storm intensity and frequency. Ecol. Model, 161, 169-182.

Lirman, D. \& Fong, P. (1996). Sequential storms cause zone-specific damage on a reef in the northern Florida reef tract: evidence from Hurricane Andrew and the 1993 Storm of the Century. Florida Sci., 59(1), 50-63.

Lirman, D. \& Fong, P. (1997a). Patterns of damage to the branching coral Acropora palmata following Hurricane Andrew: damage and survivorship of hurricane-generated asexual recruits. J. Coast. Res., 13(1), 67-72.

Lirman, D. \& Fong, P. (1997b). Susceptibility of coral communities to storm intensity, duration and frequency. Proc. $8^{\text {th }}$ Int. Coral Reef Symp., 1, 561-566.

Lluis-Riera, M. (1972). Estudios hidrológicos del Golfo de Batabanó y de las aguas oceánicas adyacentes. Serie Oceanológica, 14, 1-49.

Lluis-Riera, M. (1977). Estudios hidrológicos de la plataforma suroriental de Cuba y aguas oceánicas adyacentes. Informes Científico-Técnicos del Instituto de Oceanología, 17, 1-29.

Reshef, L., Koren, O., Loya, Y., Zilber-Rosemberg, I. \& Rosemberg, E. (2006). The coral probiotic hypothesis. Environ. Microbiol., 8(12), 2068-2073. 
Rogers, C. S. (1993). Hurricanes and coral reefs: the intermediate disturbance hypothesis revisited. Coral Reefs, 12, 127-137.

Rogers, C. S., Suchanek, T. H. \& Pecora, F. A. (1982). Effects of hurricanes David and Frederick (1979) on shallow Acropora palmata reef communities: St. Croix, US Virgin Islands. Bull. Mar. Sci., 32(2), 532-548.

Schuttenberg, H. \& Marshall, P. (2008). Managing for Mass Coral Bleaching: Strategies for Supporting Socio-ecological Resilience. En: C. Wilkinson \& D. Souter (eds.). Status of Caribbean Coral Reefs after Bleaching and Hurricanes in 2005 (pp. 115-127). Global Coral Reef Monitoring Network and Rainforest Research Centre, Townsville.
Spalding, M. D. (2004). A guide to the coral reefs of the Caribbean. University of California Press, Berkeley.

Torres, J. L. \& Morelock, J. (2002). Effect of terrigenous sediment influx on coral cover and linear extension rates of three Caribbean massive coral species. J. Caribbean Sci., 38(3-4), 222-229.

Tunnicliffe, V. (1983). Caribbean staghorn coral populations: pre-Hurricane Allen conditions in Discovery Bay, Jamaica. Bull. Mar. Sci., 33(1), 132-151.

Wilkinson, C. \& Souter, D. (2008). Status of Caribbean Coral Reefs after Bleaching and Hurricanes in 2005. Global Coral Reef Monitoring Network, and Rainforest Research Center, Townsville. 